Br Heart F 1985; 53: 43-6

\title{
Beneficial effects of diltiazem and propranolol, alone and in combination, in patients with stable angina pectoris
}

\author{
JOHN KENNY, PETER KIFF, JOANNA HOLMES, DAVID E JEWITT \\ From the Cardiac Department, King's College Hospital, London
}

SUMmARY The antianginal effects of diltiazem $180 \mathrm{mg} /$ day and propranolol $240 \mathrm{mg} /$ day, alone and in combination, were investigated in 15 patients with effort related angina in a double blind placebo controlled crossover trial, with each period of treatment lasting four weeks. Patients performed a symptom limited treadmill exercise test at the end of each period of treatment. Mean (SEM) time to onset of angina was increased from 293(32) s when receiving placebo to 347(38) s when receiving diltiazem alone, to $350(30) \mathrm{s}$ when receiving propranolol alone, and further to $421(34) \mathrm{s}$ when receiving diltiazem and propranolol combined. Similar changes occurred in the duration of exercise testing and time to $1 \mathrm{~mm}$ ST segment depression. The sum of ST segment depression at peak exercise was reduced by both diltiazem and propranolol alone compared with placebo, and combination treatment produced a further significant improvement. Rate pressure product was significantly reduced at rest and at peak exercise after propranolol alone and combination treatment. The study clearly showed the superior value of diltiazem and propranolol combined in effort related angina when compared with either drug used alone.

Until the advent of calcium channel blocking agents, beta adrenergic blocking agents in combination with nitrates had been the standard medical regimen for stable angina pectoris. Calcium channel blocking agents have proved to be an important advance in the management of ischaemic heart disease. They achieve their effect by interfering with the transmembrane transport of calcium ions in various tissues. ${ }^{1}$ Because of their ability to inhibit smooth muscle contraction they are effective in angina due to coronary artery spasm. Used alone they are also effective in chronic effort related angina pectoris and thus provide a useful form of treatment in patients in whom the use of beta adrenergic blockers is contraindicated. ${ }^{2} 3$ Several studies have shown, however, that when a calcium channel blocking agent is added to a beta adrenergic blocker in the treatment of stable angina the combi-

Requests for reprints to Dr J Kenny, Department of Medicine, Mater Misericordiae Hospital, Dublin 7, Ireland.

Accepted for publication 2 August 1984 nation is more effective than when either drug is used alone. 45

Diltiazem is a calcium channel blocking agent that has been shown to improve exercise tolerance in stable angina pectoris. ${ }^{67}$ Calcium channel blocking agents are, however, a heterogeneous group of drugs differing in both molecular structure and in their pharmacological actions. ${ }^{8}$ Diltiazem may have potential advantages over the other presently available calcium channel blocking agents. It can produce vasodilatation without increasing heart rate, has a low incidence of side effects, and can be given both intravenously and by mouth. In addition, studies in dogs have shown that it has a less negatively inotropic effect than verapamil or nifedipine. 9 Thus its effects when combined with beta blockade may differ from those of other available calcium channel blockers. We undertook this study to evaluate the effects of diltiazem when combined with propranolol in chronic exertional angina. The effects of diltiazem $180 \mathrm{mg} /$ day and propranolol $240 \mathrm{mg} / \mathrm{day}$, alone and in combination, were compared with respect to symptomatic improvement and exercise performance in 15 patients with stable angina pectoris. 


\section{Patients and methods}

Patients with a history of stable exertional angina pectoris were considered for entry into the study. Patients were included if they had chest pain associated with $>1 \mathrm{~mm}$ horizontal or down sloping ST segment depression at $0.08 \mathrm{~s}$ after the J point on treadmill exercise testing. Those who had a history of congestive cardiac failure, conduction abnormalities on the resting electrocardiogram, unstable angina pectoris, or myocardial infarction in the three months preceding the study or in whom the withdrawal of antianginal drugs was considered to be inadvisable were excluded from the study. Patients were also excluded if they had evidence of notable hepatic, renal, metabolic, or pulmonary disease.

We studied 15 men (mean (range) age 57.3 (50-67) years). Four patients had a history of transmural myocardial infarction. Coronary arteriography was performed in 14 patients. In reporting the coronary arteriogram, stenoses of the luminal diameter $\geqslant 75 \%$ were considered to be significant. Three patients had stenoses in three major vessels, five had two vessel disease, and six single vessel disease. At left ventricular angiography all patients had an ejection fraction of $>50 \%$ and a left ventricular end diastolic pressure of $<16 \mathrm{~mm} \mathrm{Hg}$.

\section{TRIAL PROTOCOL}

In this study we used a double blind placebo controlled crossover trial consisting of four periods of treatment each lasting four weeks. The four regimens were placebo, diltiazem $60 \mathrm{mg}$ three times a day, propranolol $80 \mathrm{mg}$ three times a day, and diltiazem $60 \mathrm{mg}$ combined with propranolol $80 \mathrm{mg}$ three times a day. All patients received all treatments, the order of administration being determined by a balanced latin square. The trial was double blind, and at all times the treatments were visually identical. Each period of treatment was separated by one week during which all antianginal drugs except for sublingual glyceryl trinitrate were withdrawn. At the end of a one week run in period, during which only sublingual glyceryl trinitrate was given, when required, a symptom limited treadmill exercise test was performed. Thereafter exercise tests were performed during the final week of each period of treatment and at the end of each week that separated the periods of treatment. All the treadmill exercise tests were performed according to the Bruce protocol at the same time of day for each patient. ${ }^{10}$ All exercise tests were symptom limited, the end points being severe dyspnoea, severe chest pain, or intolerable fatigue. Heart rate, blood pressure, and a modified 12 lead electrocardiogram were recorded at rest, at the end of each stage of the Bruce protocol, at peak exercise, and at intervals of two minutes during the recovery period. We were thus able to obtain exercise duration, time to $1 \mathrm{~mm} \mathrm{ST}$ segment depression, and time to the onset of angina. The electrocardiograms at each stage were analysed by calculating the sum of the number of millimetres of significant ST segment depression $(>1 \mathrm{~mm})$ in each lead at each stage. Rate pressure product was calculated as the product of systolic blood pressure and heart rate.

A subjective assessment of the efficacy of treatment during each period was obtained by patients maintaining a detailed diary of the frequency of anginal attacks and the number of sublingual tablets of glyceryl trinitrate consumed. Sublingual glyceryl trinitrate was not permitted prophylactically.

\section{STATISTICAL ANALYSIS}

Statistical analysis was performed with analysis of variance of repeated measurements. ${ }^{11}$ If a significant result was found, Student's paired $t$ test was used for comparison between two treatments. All values were expressed as mean (SEM).

\section{Results}

\section{SUBJECTIVE DATA}

Although the total number of anginal episodes a week was reduced from $7(3)$ during treatment with placebo to 3(1) during treatment with diltiazem, 2(1) during treatment with propranolol, and 1(1) during combination treatment, this reduction was not significant. Similarly, glyceryl trinitrate consumption per week was not significantly decreased, though it was reduced from 6(3) during treatment with placebo to 3(1) during treatment with diltiazem, $1(1)$ during treatment with propranolol, and $1(1)$ during combination treatment.

\section{OBJECTIVE DATA}

Combination treatment with diltiazem and propranolol produced significant increases in the duration of exercise, time to the onset of angina, and time to $1 \mathrm{~mm}$ ST depression compared with placebo (Table 1). Duration of exercise increased from $371(39) \mathrm{s}$ during treatment with placebo to $437(28) \mathrm{s}$ during combination treatment $(p<0.02)$. Duration of exercise improved significantly compared with placebo during propranolol treatment, but no significant change occurred during diltiazem treatment. Time until the onset of angina or until the stopping of exercise if angina did not develop increased from 293(32) s with placebo to 421(34) s $(p<0.001)$ during treatment with diltiazem and propranolol combined. A similar significant increase of the order of $40 \%$ occurred in the duration of exercise to the onset of $1 \mathrm{~mm}$ ST segment depression after com- 
Beneficial effects of diltiazem and propranolol, alone and in combination, in patients with stable angina pectoris 45

Table 1 Mean (SEM) total duration of exercise, time to onset of angina, and time to $1 \mathrm{~mm} S T$ segment depression in 15 patients receiving four different treatments

\begin{tabular}{lllll}
\hline & Placebo & Diltiazem & Propranolol & Diliazem and propranolol \\
\hline Duration of exercise (s) & $371(39)$ & $396(32)$ & $412(29)^{\star}$ & $437(28)^{\star \star}$ \\
Time to angina (s) & $293(32)$ & $347(38)^{\star \star \star}$ & $350(30)^{\star \star \star}$ & $421(34)^{\star \star \star \star}$ \\
Time to 1 mm ST depression (s) & $292(37)$ & $332(37)^{\star}$ & $387(37)^{\star \star \star}$ & $403(34)^{\star \star \star}$ \\
\hline
\end{tabular}

Significance of difference when compared with placebo: ${ }^{\star} p<0.05 ;{ }^{\star \star} p<0.02 ;{ }^{\star \star \star} p<0.01 ;{ }^{\star \star \star \star} p<0.001$.

Table 2 Mean (SEM) rate-pressure product at rest and peak exercise in 15 patients receiving four different treatments

\begin{tabular}{lllll}
\hline & Placebo & Diltiazem & Propranolol & Diltiazem and propranolol \\
\hline $\begin{array}{l}\text { Rate-pressure product }(\mathrm{mm} \mathrm{Hg} \\
\text { beat } / \text { min } \times 10^{-3} \text { ) at: }\end{array}$ & & & \\
$\begin{array}{l}\text { Rest } \\
\text { Peak exercise }\end{array}$ & $12 \cdot 1(0.6)$ & $10 \cdot 8(0.9)$ & $7 \cdot 5(0.4)^{\star}$ & $6 \cdot 3(0.6)^{\star}$ \\
\hline
\end{tabular}

Significance of difference when compared with placebo: ${ }^{\star} p<0.001$.

bination treatment compared with placebo.

Treatment with either diltiazem or propranolol alone produced significant increases in time to onset of angina and $1 \mathrm{~mm}$ ST depression, but these increases were not as pronounced as those occurring during combination treatment. Angina pectoris occurred during the treadmill exercise test in all patients when receiving placebo. Angina was absent in seven patients when receiving combination treat: ment ( $p<0.05$ compared with placebo; McNemar's test). Angina pectoris was absent in four patients when receiving diltiazem and two when receiving propranolol.

Reflecting reduced electrocardiographic evidence of ischaemia, the sum of ST segment depression at peak exercise decreased significantly from $6.7(0.9) \mathrm{mm}$ during placebo treatment to $3.9(0.7) \mathrm{mm}$ during combination treatment $(p<0.01)$. A significant reduction, to $5.4(0.9) \mathrm{mm}(\mathrm{p}<0.05)$, also occurred during propranolol treatment, but treatment with diltiazem produced only a non-significant reduction to $6 \cdot 1(0 \cdot 9)$ $\mathrm{mm}$. Rate pressure product fell during combination treatment by about $50 \%$ when compared with placebo (Table 2). Similarly, at peak exercise rate, pressure product decreased from $23.7(1.5) \mathrm{mm} \mathrm{Hg}$ beats $/ \mathrm{min}$ $10^{-3}$ with placebo to $14.0(0.8) \mathrm{mm} \mathrm{Hg}$ beats $/ \mathrm{min} 10^{-3}$ during combination treatment $(\mathrm{p}<0.001)$. Propranolol alone, but not diltiazem, produced significant reductions in rate pressure product at rest and during exercise.

\section{ADVERSE EFFECTS}

No important adverse effects were noted in patients taking placebo, diltiazem, or propranolol. Adverse effects were noted in two patients who received a combination of diltiazem and propranolol. One, who had a previous anterior myocardial infarct and a dyskinetic anterolateral segment at left ventricular angiography, developed dyspnoea after starting combination treatment. This was associated with a sinus bradycardia of 45 beats/minute and resolved on stopping treatment. The second patient developed fatigue associated with a sinus bradycardia of 36 beats/ minute. This adverse effect responded to a reduction by one tablet in the dose of each drug: diltiazem dosage was reduced to $120 \mathrm{mg} /$ day and propranolol to 160 $\mathrm{mg} /$ day.

\section{Discussion}

The beneficial effect in angina pectoris of combined diltiazem and propranolol treatment has been shown in this study by the objective evidence of increased duration of exercise, increased time to the onset of angina, and increased time to $1 \mathrm{~mm} \mathrm{ST} \mathrm{segment}$ depression. This was coupled with reduced electrocardiographic evidence of ischaemia compared with placebo. Although there was a trend towards subjective improvement as evidenced by the frequency of angina and the consumption of glyceryl trinitrate, this change was not significant.

The objective evidence of improvement supports similar studies showing that, when calcium channel blocking agents such as nifedipine or verapamil are combined with a beta blocker, combination treatment is more effecive that either a calcium channel blocker or a beta blocker used alone. ${ }^{45}$ Diltiazem alone has been shown to improve exercise tolerance in angina pectoris, and this effect appears to be more pronounced with increasing dosages. ${ }^{6712}$ In this study we used diltiazem in a dose of $180 \mathrm{mg} / \mathrm{day}$, at which dose it did not produce a significant improvement in duration of exercise or electrocardiographic evidence of ischaemia. Some dose titration studies have shown little benefit in exercise tolerance when diltiazem is used in doses of less than $240 \mathrm{mg} /$ day. ${ }^{613}$ In a recent 
study similar in design to ours diltiazem in a dose of $360 \mathrm{mg} /$ day appeared to be as effective or more effective than combined diltiazem ( $360 \mathrm{mg} /$ day) and propranolol $\left(240 \mathrm{mg} /\right.$ day).$^{14}$ In that study, combination treatment when compared with diltiazem alone, although not prolonging exercise time, did reduce electrocardiographic evidence of ischaemia. Patients in the study on combination treatment were often limited by fatigue. Similarly, in our study, although time to onset of angina was increased by $40 \%$, the duration of exercise was increased by only $18 \%$ compared with placebo as many patients, although not experiencing angina, were limited by fatigue. This was probably due to an appreciable reduction in peak heart rate. In the study of Hung et al four of 12 patients receiving combination treatment experienced side effects requiring a reduction in dosage compared with two of 15 patients in our study.

The beneficial action of calcium channel blockers in effort induced angina is multifactorial. ${ }^{1}$ They may increase myocardial oxygen supply by increasing coronary blood flow. They may also reduce myocardial oxygen demand by reducing afterload by decreasing peripheral vascular resistance, and in some cases reducing contractility and heart rate. Combination treatment with diltiazem and propranolol produced significant reductions of the order of $40 \%$ in rate pressure product both at rest and peak exercise. Rate pressure product during exercise has been shown to correlate well with myocardial oxygen consumption. ${ }^{15}$ Thus combination treatment appears to achieve its beneficial effect through a reduction in myocardial oxygen demand. Propranolol alone also significantly reduced rate pressure product both at rest and during exercise but not to the same extent as combination treatment. Diltiazem alone did not significantly alter rate pressure product, and this may well account for its failure to improve duration of exercise and reduce electrocardiographic evidence of ischaemia. Nevertheless, it increased time to the onset of angina and time to $1 \mathrm{~mm} \mathrm{ST} \mathrm{depression.} \mathrm{This} \mathrm{beneficial} \mathrm{effect} \mathrm{may}$ have been related to increased myocardial oxygen supply as diltiazem has been shown to increase coronary sinus blood flow in man. ${ }^{16}$

This study objectively shows the superior value of diltiazem and propranolol combined in effort related angina compared with either drug used alone. The beneficial effects of combination treatment appear to be mediated by a reduction in myocardial oxygen consumption. Caution in using combined diltiazem and propranolol treatment is, however, indicated by this and other studies, and careful titration of diltiazem dosage should be carried out to minimise side effects.

\section{References}

1 Braunwald E. Mechanism of action of calcium-channelblocking agents $N$ Engl $\mathcal{Y}$ Med 1982; 307: 1618-27.

2 Balasubramanian V, Paramasivan R, Lahiri A, Raftery EB. Verapamil in chronic stable angina. Lancet 1980; i: 841-4.

3 Mueller HS, Chahine RA. Interim report of multicenter double-blind, placebo controlled studies of nifedipine in chronic stable angina. Am $\mathcal{F}$ Med 1981; 71: 645-57.

4 Subramanian B, Bowles MJ, Davies AB, Raftery EB. Combined therapy with verapamil and propranolol in chronic stable angina. Am $\mathcal{F}$ Cardiol 1982; 49: 125-32.

5 Lynch P, Dargie H, Krikler S, Krikler D. Objective assessment of antianginal treatment: a double-blind comparison of propranolol, nifedipine and their combination. $\mathrm{Br} \mathrm{Med} F$ 1980; 281: 184-7.

6 Hossack KF, Bruce RA, Trimble S, Kusumi F. Improved exercise performance in patients with stable angina pectoris receiving diltiazem. Am f Cardiol 1981; 47: 95-101.

7 Hossack KF, Pool PE, Steele P, et al. Efficacy of diltiazem in angina on effort: a multicenter trial. Am $\mathcal{F}$ Cardiol 1982; 49: 567-72.

8 Henry PD. Comparative pharmacology of calcium antagonists: nifedipine, verapamil and diltiazem. $A m \mathcal{F}$ Cardiol 1980; 46: 1047-58.

9 Walsh RA, Badke FR, O'Rourke RA. Differential effects of systemic and intracoronary calcium channel blocking agents on global and regional left ventricular function in conscious dogs. Am Heart $\mathcal{F}$ 1981; 102: 34150.

10 Bruce RA, Hornsten TR. Exercise stress testing in evaluation of patients with ischemic heart disease. Prog Cardiovasc Dis 1969; 11: 371-90.

11 Winer BJ. Statistical principles in experimental design. 2nd ed. New York: McGraw Hill, 1971:197-200.

12 Subramanian VB, Khurmi NS, Bowles MJ, O'Hara M, Raftery EB. Objective evaluation of three dose levels of diltiazem in chronic stable angina. $\mathcal{F ~ A m ~ C o l l ~ C a r d i o l ~}$ 1983; 1: 114-53.

13 Low RI, Takeda P, Lee G, Mason DT, Awan NA, DeMaria AN. Effects of diltiazem-induced calcium blockade upon exercise capacity in effort angina due to chronic coronary artery disease. Am Heart $\mathcal{F}$ 1981; 101: 713-8.

14 Hung J, Lamb IH, Connolly SJ, Jutzy KR, Goris ML, Schroeder JS. The effect of diltiazem and propranolol, alone and in combination, on exercise performance and left ventricular function in patients with stable effort angina: a double-blind, randomized, and placebocontrolled study. Circulation 1983; 68: 560-7.

15 Holmberg S, Serzysko W, Varnauskas E. Coronary circulation during heavy exercise in control subjects and patients with coronary heart disease. Acta Med Scand 1971; 190: 465-80.

16 Bourassa MG, Cote P, Theroux P, Tubau JF, Genain C, Waters DD. Hemodynamics and coronary flow following diltiazem administration in anesthetised dogs and in humans. Chest 1980; 78: 224-30. 